\title{
Active Imaging with Dual Spin-Echo Diffusion MRI
}

\author{
Jonathan D. Clayden ${ }^{1}$, Zoltan Nagy ${ }^{2}$, Matt G. Hall ${ }^{3,4}$, Chris A. Clark ${ }^{1}$, and \\ Daniel C. Alexander ${ }^{3,4}$ \\ 1 Institute of Child Health \\ 2 Wellcome Trust Centre for Neuroimaging \\ 3 Department of Computer Science \\ 4 Centre for Medical Image Computing, University College London, UK \\ E-mail: j.clayden@ucl.ac.uk
}

\begin{abstract}
Active imaging is a recently developed approach to modelbased optimisation of imaging protocols. In the application we discuss here, a diffusion magnetic resonance imaging (dMRI) protocol is optimised for directly measuring aspects of biological tissue microstructure, subject to appropriate scanner hardware and acquisition time constraints. We present the theoretical basis for active imaging with the dual spin-echo (DSE) dMRI pulse sequence, which is more complex than the standard sequence, but widely used due to its robustness to image distortion. The new formulation provides the basis for future active imaging studies using DSE. To demonstrate the approach, we optimise DSE sequences for estimating parameters in a simple model of neural white matter, specifically axon density and diameter. Results show that sensitivity to these important parameters is at least as good as with more traditional pulse sequences that are not robust to image distortion.
\end{abstract}

\section{Introduction}

Diffusion magnetic resonance imaging (dMRI) is an umbrella term for a set of magnetic resonance acquisition and processing techniques in which MRI is sensitised to the random movement of particles in a sample or subject [1]. In living subjects, the diffusing species is almost invariably water. By characterising the diffusion process, information about the microstructure and connectivity of the underlying tissue can be obtained; and in the brain, in particular, dMRI has proven to be a practical and versatile tool for clinical and neuroscientific work. By applying diffusion sensitivity along one or more directions, diffusion weighted images are produced, and these have been particularly valuable in the study of stroke [2]. However, the later development of diffusion tensor imaging (DTI) [3] has broadened the usefulness of dMRI considerably. DTI uses diffusion weighted measurements along at least six noncollinear directions to estimate a rank-two diffusion tensor at each point in the image, which is proportional to the covariance matrix of a 3D Gaussian distribution over molecular displacements. A scalar value commonly derived from the tensor is fractional anisotropy, a 
measure of dispersion in the tensor eigenvalues, which has been shown to be locally or globally reduced in a number of diseases [4], as well as some psychiatric disorders such as schizophrenia [5], reflecting changes in the underlying tissue. The principal eigenvector of the tensor, indicating the direction of greatest meansquared displacement, was the original basis for dMRI-based white matter fibre tracking, which has provided insights in basic neuroscience with regard to the connectivity of the brain (e.g. [6]).

As useful as simple, DTI-derived measures of diffusion magnitude, direction and anisotropy have proven to be, they do not provide specific information about tissue microstructure. In reality, several distinct populations of water molecules, both inside and outside of neural cells, contribute to the dMRI signal, and so the displacement distribution is more complex than the Gaussian model used in DTI. However, analytical expressions for the displacement distribution and dMRI signal in a number of regular, bounded geometries are now well established [7], allowing more sophisticated approaches to construct geometric tissue models and combine these expressions to obtain better signal models $[8,9]$. These models potentially include a wide variety of parameters relating directly to microstructure, such as the radius and density of neuronal axons and other cellular structures, diffusivities in various parts of the tissue, permeabilities of different cell types, myelin thickness or the relative proportions of white matter and other tissue types in the imaged region - since all of these parameters affect water mobility and thus the dMRI signal. This exciting new area of development for dMRI offers the possibility of measuring these parameters in live tissue, thereby providing new insight into the workings of the brain and the mechanisms of disease, as well as important new biomarkers.

Previous work has demonstrated the viability of estimating microstructure parameters both in simulation and in real tissue [10-12]. For example, Stanisz et al. [11] used a white matter model incorporating two cell types, axons (modelled with prolate ellipsoids) and glia (spheres), with permeable membranes; and they fitted their model to image data acquired from excised optic nerve tissue. More recently, Assaf et al. [10] used a model incorporating cylindrical axons with radii following a gamma distribution, whose parameters were fitted to data from ex vivo optic and sciatic nerves. The imaging protocols in these studies are, however, impractical for use on live humans, due to the large gradient strengths and scan times required: the former up to $1400 \mathrm{mT} \mathrm{m}^{-1}$ (compared to the $32 \mathrm{mT} \mathrm{m}^{-1}$ available on a typical clinical scanner), and the latter often on the order of many hours to days. Moreover, they exploit a priori knowledge of white matter tract orientation, which is not generally available in vivo.

Active imaging optimises imaging protocols for sensitivity to specific parameters of a tissue model, subject to scan hardware and time constraints [13,14]. The term "active imaging" comes from active learning, which is a branch of machine learning that aims to identify the minimum set of enquiries required to determine the state of a system [15]. Optimising an imaging paradigm for estimating tissue microstructure parameters reduces to the same problem. In [16], an active imaging algorithm is constructed for optimising dMRI protocols 
when the fibre orientation is unknown. Simulations using a signal model similar to that in [10] demonstrate the feasibility of measuring axon size and density in live human subjects on current systems. The technique generalises to more complex models incorporating a variety of useful microstructural parameters and provides the opportunity, for the first time, to map these parameters over the live human brain.

A limitation of the approach in [16] is that it assumes the dMRI measurements come from the standard pulsed-gradient spin-echo (PGSE) pulse sequence. Here, we extend the method to the more complex dual spin-echo (DSE) sequence, which is more useful in practice as it is more robust to distortion effects. In $\S 2$ we give some background on how MRI is sensitised to diffusion, and on microstructural models of the dMRI signal. In $\S 3$ we develop a full parameterisation of the DSE pulse sequence, and establish bounds on each parameter; we extend a previous model of the dMRI signal arising from cylindrical axons to the DSE case; and we integrate the new encoding into the optimisation framework in [16]. In $\S 4$, we demonstrate the approach, comparing optimised DSE and PGSE protocols for estimating axon density and diameter in simulation. We conclude in $\S 5$. This study provides the foundational techniques for the future development of DSE pulse sequences optimised for any suitable tissue model, with recovered parameters as specific as the data admit.

\section{Background}

In this section we provide relevant background material on dMRI pulse sequences and the nature of diffusion in white matter.

\subsection{Pulse Sequences}

There are a number of ways of using magnetic gradient pulses to achieve diffusion sensitivity in an MRI experiment. The original PGSE sequence was described by Stejskal \& Tanner [17], and is shown schematically in Fig. 1a. It consists of a pair of gradient pulses of equal magnitude and length $\delta$, either side of a radio frequency $(\mathrm{RF})$ inversion, or $180^{\circ}$, pulse. The first gradient applies a spatially varying phase shift to diffusing spins, which is reversed by the second pulse if the molecules do not move. However, molecular movement results in a residual phase offset, which manifests as an attenuation in the signal. The greater the aggregate distance moved due to diffusion along the direction of the gradient, the more greatly attenuated is the signal measured.

Unfortunately this sequence has a practical problem: electric eddy currents are induced by the onset and offset of the gradient pulses, causing distortion effects in the acquired image data. Some of these effects decay with time constants comparable to the length of the sequence, and so in practice they tend to accumulate. The recently developed DSE sequence [18] - shown in Fig. 1bworks similarly, but has much improved robustness to eddy current effects. In fact, the sequence can be configured to eliminate entirely eddy current effects 

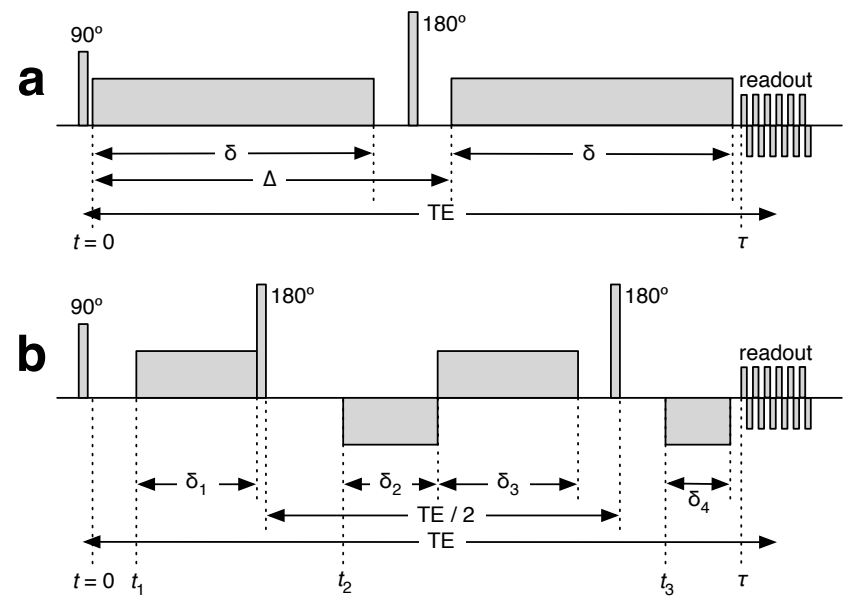

Fig. 1. Schematic representations of the standard pulsed-gradient spin-echo (a) and dual spin-echo (b) dMRI pulse sequences. The axis represents time.

with a particular time constant. For this reason, it is becoming the standard diffusion sequence on commercial MRI scanners. In DSE, the initial phase shift is provided by a combination of the first two gradient pulses, with lengths $\delta_{1}$ and $\delta_{2}$. (These pulses appear with opposite sign in the figure, but the phase effect of all gradients is inverted between the two $180^{\circ} \mathrm{RF}$ pulses.) Rephasing is effected by the remaining two pulses, and there is therefore a "balance" requirement that

$$
\delta_{1}+\delta_{2}=\delta_{3}+\delta_{4} .
$$

Since large gradient magnitudes are usually required for measuring tissue microstructure features, and eddy current effects scale with gradient strength, the DSE sequence is particularly appealing for this application. However, the sequence has not previously been used for estimating tissue model parameters, due to the relative difficulty of designing by hand a suitable protocol based on this more complex sequence, combined with an absence of analytical signal models. This paper provides an encoding of the DSE sequence, enabling optimisation of the protocol with active imaging. We also derive models of the DSE signal from water in simple restricted geometries, which allows us to extend the microstructural dMRI models in $[10,11,16]$ for DSE. In combination, these novel contributions enable microstructure imaging protocols free of image distortion, which is an essential step towards their realisation in practice.

\subsection{Hindered and Restricted Diffusion}

Under the simple model used in [8], neural white matter tissue consists of two compartments: a homogeneous substrate, and a set of impermeable cells with 
regular geometry embedded within the substrate. Diffusion within the cells is therefore restricted by their geometry, while extracellular diffusion is merely hindered. In common with the CHARMED model [9], it is assumed that the total normalised signal, $E$, arising from the two compartments is a linear combination of contributions from each, viz.

$$
E(\mathbf{G}, \Theta, \Phi)=f E_{r}(\mathbf{G}, \Theta, \Phi)+(1-f) E_{h}(\mathbf{G}, \Theta, \Phi),
$$

where $f \in[0,1]$ is the volume fraction of the intracellular compartment, $\mathbf{G}$ is the diffusion gradient direction, $\Theta$ is a set of sequence parameters, and $\Phi$ is a set of parameters for the tissue model.

The signal due to hindered diffusion, $E_{h}$, can be modelled by assuming a standard Gaussian displacement distribution, as in DTI. Diffusion in the restricted compartment is far from Gaussian, however, and depends on the geometry of the cells. By solving the diffusion equation subject to suitable boundary conditions, Neuman [7] derives expressions for the displacement distributions in spheres and cylinders. He then calculates approximations for the signal, $E_{r}$, within these restricted domains in the presence of a single continuous gradient, based on the assumption of a Gaussian distribution over phases among the diffusing molecules. The equivalent expression for cylindrical domains with the PGSE sequence was given by van Gelderen [19], and we give the new DSE solution below.

\section{Methods}

This section begins with our parameterisation of the DSE pulse sequence. We then derive a model for the restricted signal arising from a dMRI experiment under this parameterisation.

\subsection{Parameterisation and Constraints}

Figure $1 \mathrm{~b}$ shows the DSE pulse sequence. It involves four individual diffusionsensitising pulses of lengths $\delta_{1}, \delta_{2}, \delta_{3}$ and $\delta_{4}$, split into three blocks separated by two $180^{\circ} \mathrm{RF}$ pulses. The first gradient pulse occurs at a time $t_{1}$ after the initial excitation $\left(90^{\circ}\right) \mathrm{RF}$ pulse. The second occurs at time $t_{2}$, the third immediately after the second, and the fourth at time $t_{3}$. Additionally, we choose to arrange that the first $180^{\circ}$ pulse occurs immediately after the first gradient pulse.

The diffusion sensitisation pulses cannot take up the whole echo time (TE) of a DSE sequence, because time is required for the $180^{\circ}$ pulses; and for excitation and "readout", or signal measurement, at the beginning and end of the sequence respectively. We denote the total amount of time actually available for the diffusion pulses with $\tau=\mathrm{TE}-T_{\text {prep }}-T_{\text {read }}$, and the time required for each $180^{\circ}$ pulse with $P_{180}$. The time between the two $180^{\circ}$ pulses, within which the second and third diffusion gradients must fit, is exactly $T_{\text {inner }}=\mathrm{TE} / 2-P_{180}$. The time available for the outer two pulses is therefore $T_{\text {outer }}=\tau-\mathrm{TE} / 2-P_{180}$. It should be noted that as TE increases, the basic signal-to-noise ratio (SNR) of the sequence will decrease because of spontaneous dephasing due to spin relaxation. 
We encode the sequence with the parameter set $\Theta=\left\{\mathrm{TE}, T, t_{s}, \delta_{1}, t_{1}, t_{2}, t_{3}, G\right\}$, where $T=\delta_{1}+\delta_{2}+\delta_{3}+\delta_{4}$ and $t_{s}=\delta_{2}+\delta_{3}-\delta_{1}-\delta_{4}$. We include $T$ and $t_{s}$ rather than $\delta_{2}$ and $\delta_{3}$ for convenience when nulling eddy currents in $\S 3.2$. The fourth pulse length need not be included because of the balancing constraint in (1). A number of constraints on these parameters must be satisfied in order to ensure that the diffusion weighting properties of the sequence remain intact, and that all pulse lengths are nonnegative. These are outlined below.

The time available for the outer two pulses, $\delta_{1}$ and $\delta_{4}$, must be nonnegative; and so it follows directly from the definitions of $T_{\text {outer }}$ and $\tau$ above that

$$
\mathrm{TE} \geq 2\left(P_{180}+T_{\text {prep }}+T_{\text {read }}\right) .
$$

Due to the balancing constraint, we can write $T=2\left(\delta_{1}+\delta_{2}\right)$, which has bounds

$$
0 \leq T \leq \mathrm{TE}-2\left(P_{180}+T_{\text {prep }}+T_{\text {read }}\right)=2 \tau-\mathrm{TE}-2 P_{180} .
$$

It follows from the balancing constraint and the definitions of $T$ and $t_{s}$ that $T+t_{s}=2\left(\delta_{2}+\delta_{3}\right)$, which is bounded by the time available for the inner two pulses. Similarly, $T-t_{s}=2\left(\delta_{1}+\delta_{4}\right)$, so we have

$$
T+t_{s} \leq 2 T_{\text {inner }} \text { and } T-t_{s} \leq 2 T_{\text {outer }} .
$$

Moreover, since no single diffusion pulse can be longer than $T / 2$, or negative in length, the absolute difference between $\delta_{3}$ and $\delta_{1}$ also cannot exceed $T / 2$. As a result, $\left|t_{s}\right|$ cannot exceed $T$. The full bounds on $t_{s}$ are therefore

$$
\max \left\{-T, \mathrm{TE}-2 \tau+2 P_{180}+T\right\} \leq t_{s} \leq \min \left\{T, \mathrm{TE}-2 P_{180}-T\right\} .
$$

Given valid choices of $T$ and $t_{s}$, subject to these bounds, we will be able to recover the lengths of the second and third pulses as

$$
\delta_{2}=T / 2-\delta_{1} \quad \text { and } \quad \delta_{3}=t_{s} / 2+\delta_{1},
$$

subject to first choosing $\delta_{1}$. The latter must be constrained such that none of the other three pulse lengths will be negative:

$$
\max \left\{0,-t_{s} / 2\right\} \leq \delta_{1} \leq \min \left\{T / 2,\left(T-t_{s}\right) / 2\right\} .
$$

The times of onset are also constrained. They must all be nonnegative, and must leave room for each pulse. The upper bound on $t_{3}$ is set by the requirement that the last pulse must finish before $t=\tau$, but it must occur after the second refocussing pulse. We therefore have

$$
\begin{aligned}
0 & \leq t_{1} \leq T_{\text {outer }}-2 \delta_{1}-\delta_{2}+\delta_{3} ; \\
t_{1}+\delta_{1}+P_{180} & \leq t_{2} \leq t_{1}+\delta_{1}+\mathrm{TE} / 2-\delta_{2}-\delta_{3} ; \\
t_{1}+\delta_{1}+P_{180}+\mathrm{TE} / 2 & \leq t_{3} \leq \tau-\left(\delta_{1}+\delta_{2}-\delta_{3}\right) .
\end{aligned}
$$

The final relevant parameter is $G$, the magnitude of the gradient pulses, but this is independent of the other quantities and bounded simply by $0 \leq G \leq G_{\max }$, for some $G_{\max }$ appropriate to the scanner being used. All four gradient pulses in each arrangement use the same $G$. 


\subsection{Nulling Eddy Current Effects}

The ability to null eddy current induced distortion effects is the major benefit of DSE over PGSE. Heid [20] established that eddy current effects proportional to $e^{-\lambda_{0} t}$ will vanish exactly if

$$
\delta_{1}=\frac{1}{\lambda_{0}} \ln \left(\frac{1+\cosh \frac{\lambda_{0} T}{2}}{\exp \frac{\lambda_{0} t_{s}}{2}+\exp \frac{-\lambda_{0} T}{2}}\right) .
$$

Off-design eddy current effects, with time constants different from $\lambda_{0}$, will also be reduced, although not totally nulled. We can substitute this fixed form for the bounds given by (8), thus improving the eddy current distortion properties of the sequence further, at the cost of losing one degree of freedom in the parameterisation. In this case, the bounds on $T, t_{1}, t_{2}$ and $t_{3}$ remain exactly as before, although the limits on $t_{s}$ take a different form. The right hand side of (12) must be nonnegative, so we constrain

$$
t_{s} \leq \frac{2}{\lambda_{0}} \ln \left(1+\sinh \frac{\lambda_{0} T}{2}\right) .
$$

In addition, we observe from (7) that $\delta_{2}$ is negative unless $\delta_{1} \leq T / 2$. This condition imposes the additional constraint

$$
t_{s} \geq \frac{2}{\lambda_{0}} \ln \left(\frac{1+e^{-\lambda_{0} T}}{2}\right) .
$$

The limits described by (5) also continue to apply.

\subsection{Signal Model}

The model of neural white matter that we use here to demonstrate active imaging with DSE consists of nonabutting cylinders of radius $R$, representing neural axons, in a homogeneous substrate [8]. The signal model takes the form of (2), assuming cylindrically symmetric Gaussian diffusion in the hindered, extracellular compartment. Here we derive an expression for the DSE signal in the intracellular compartment.

We decompose the intracellular signal into components parallel and perpendicular to the axons. In the parallel case, diffusion is assumed to be univariate Gaussian with diffusivity $d_{\|}$; and the effective gradient strength is $G \cos \beta$, where $\beta$ is the angle between the gradient direction, $\mathbf{G}$, and the fibre direction, $\mathbf{n}$. The perpendicular component, however, exhibits restricted diffusion due to the inner walls of the axons. Following Neuman [7], assuming a Gaussian distribution of phases in the tissue, we integrate over molecular displacements during the course of the sequence to arrive at the form

$$
E_{r \perp}(\mathbf{G}, \Theta, \Phi)=-2 \gamma^{2}(G \sin \beta)^{2} \sum_{m=1}^{\infty} \frac{\zeta_{m}}{\alpha_{m}^{6} d_{\perp}^{2}\left(\alpha_{m}^{2} R^{2}-1\right)},
$$


where

$$
\begin{gathered}
\zeta_{m}=2 \alpha_{m}^{2} d_{\perp}\left(\delta_{1}+\delta_{2}\right)-\left[5+Y_{m}\left(t_{2}-t_{1}\right)-Y_{m}\left(t_{3}-t_{1}\right)-Y_{m}\left(t_{3}-t_{2}\right)-Y_{m}\left(\delta_{1}\right)\right. \\
-Y_{m}\left(t_{2}-t_{1}-\delta_{1}\right)+Y_{m}\left(t_{3}-t_{1}-\delta_{1}\right)-2 Y_{m}\left(\delta_{2}\right)-2 Y_{m}\left(t_{2}-t_{1}+\delta_{2}\right) \\
+2 Y_{m}\left(t_{2}-t_{1}+\delta_{2}-\delta_{1}\right)+2 Y_{m}\left(t_{3}-t_{2}-\delta_{2}\right)-2 Y_{m}\left(\delta_{3}\right)+Y_{m}\left(\delta_{2}+\delta_{3}\right) \\
+Y_{m}\left(t_{2}-t_{1}+\delta_{2}+\delta_{3}\right)-Y_{m}\left(t_{2}-t_{1}+\delta_{2}+\delta_{3}-\delta_{1}\right) \\
-2 Y_{m}\left(t_{3}-t_{2}+\delta_{1}-\delta_{3}\right)-Y_{m}\left(t_{3}-t_{1}+\delta_{2}-\delta_{3}\right)-Y_{m}\left(\delta_{1}+\delta_{2}-\delta_{3}\right) \\
+Y_{m}\left(t_{3}-t_{1}+\delta_{1}+\delta_{2}-\delta_{3}\right)+Y_{m}\left(t_{3}-t_{2}+\delta_{1}+\delta_{2}-\delta_{3}\right) \\
\left.-Y_{m}\left(t_{3}-t_{2}-\delta_{2}-\delta_{3}\right)+Y_{m}\left(t_{3}-t_{2}+\delta_{1}-2 \delta_{3}\right)\right]
\end{gathered}
$$

and

$$
Y_{m}(x)=\exp \left(-\alpha_{m}^{2} d_{\perp} x\right) .
$$

The term $\alpha_{m}$ represents the $m$ th smallest $\alpha$ solving the equation $J_{1}^{\prime}(\alpha R)=0$, where $J_{1}^{\prime}$ is the derivative of the Bessel function of the first kind, order one. The total signal from the restricted compartment is finally

$$
E_{r}(\mathbf{G}, \Theta, \Phi)=E_{r \|}(\mathbf{G}, \Theta, \Phi) E_{r \perp}(\mathbf{G}, \Theta, \Phi) .
$$

The full set of model parameters is therefore $\Phi=\left\{f, R, \mathbf{n}, d_{\|}, d_{\perp}\right\}$.

\section{Experiments and Results}

In all our experiments, we use parameter settings similar to the natural values in human white matter, and sequence constraints which are easily achievable on most clinical MRI scanners. Specifically, we choose $f=0.7, d_{\|}=1.7 \times$ $10^{-9} \mathrm{~m}^{2} \mathrm{~s}^{-1}$ and $d_{\perp}=1.2 \times 10^{-9} \mathrm{~m}^{2} \mathrm{~s}^{-1}$. We take $G_{\max }=32 \mathrm{mT} \mathrm{m}^{-1}$ and assume a base SNR of 10 at $\mathrm{TE}=90 \mathrm{~ms}$ - both of which are well within reach of most scanners.

We constrain the protocol to contain $M$ different sets of sequence parameters, each of which are applied along $N$ noncollinear gradient directions. We use the optimisation framework and cost function described in [16], based on the Cramér-Rao Lower Bound (CRLB) and a Rician noise model, which is appropriate for MRI images [21]. The noise level used to calculate the cost function is adjusted in line with the optimised TE, as in [16], which we choose to fix over all pulse arrangements, leaving $7 M+1$ parameters to optimise over. Again following [16], we use $M=4$ and $N=90$ throughout, for a total of 360 measurements, which is achievable in under an hour with a modern human system.

The first experiment compares the sensitivity of the PGSE and DSE sequences to the parameters of the tissue model, as indicated by the cost function itself. We optimise both sequences for each $R \in\{1,3,5,10,20\} \mu$ m separately. The DSE sequence is optimised both without and with the eddy current nulling constraint in (12). For the latter, we set $\lambda_{0}=0.7 / \tilde{T}$ as in [20], where $\tilde{T}$ is the maximal $T$ across all pulse arrangements. The means and coefficients of variation $(\mathrm{CVs})$ of the cost function over 500 fibre orientations equally distributed on the 

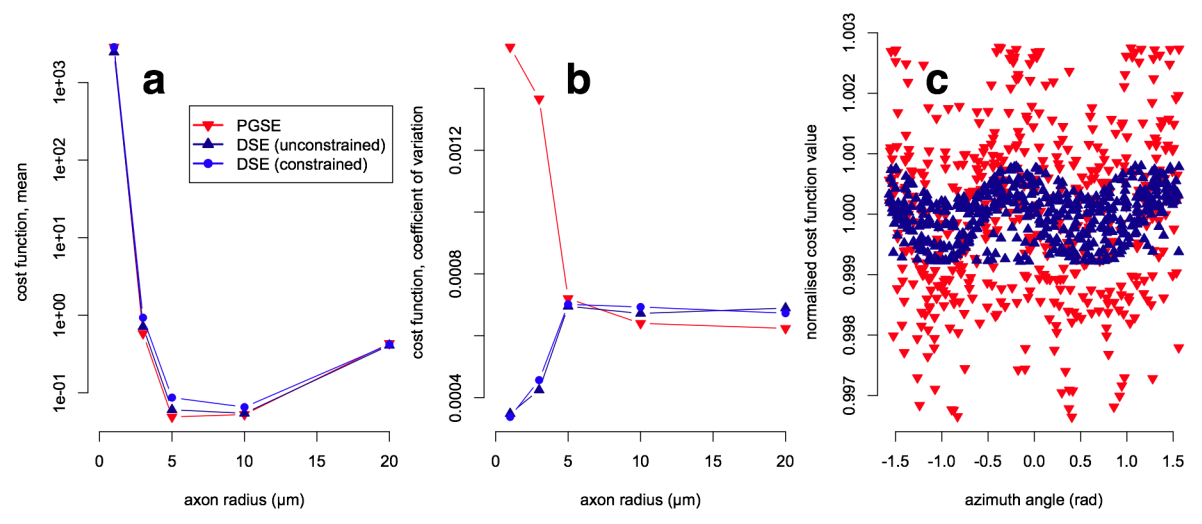

Fig. 2. Trends in the cost function with axon radius $(a, b)$ and azimuth angle (c). $R=3 \mu \mathrm{m}$ in $\mathrm{c}$; base $\mathrm{SNR}=10$ in all cases.
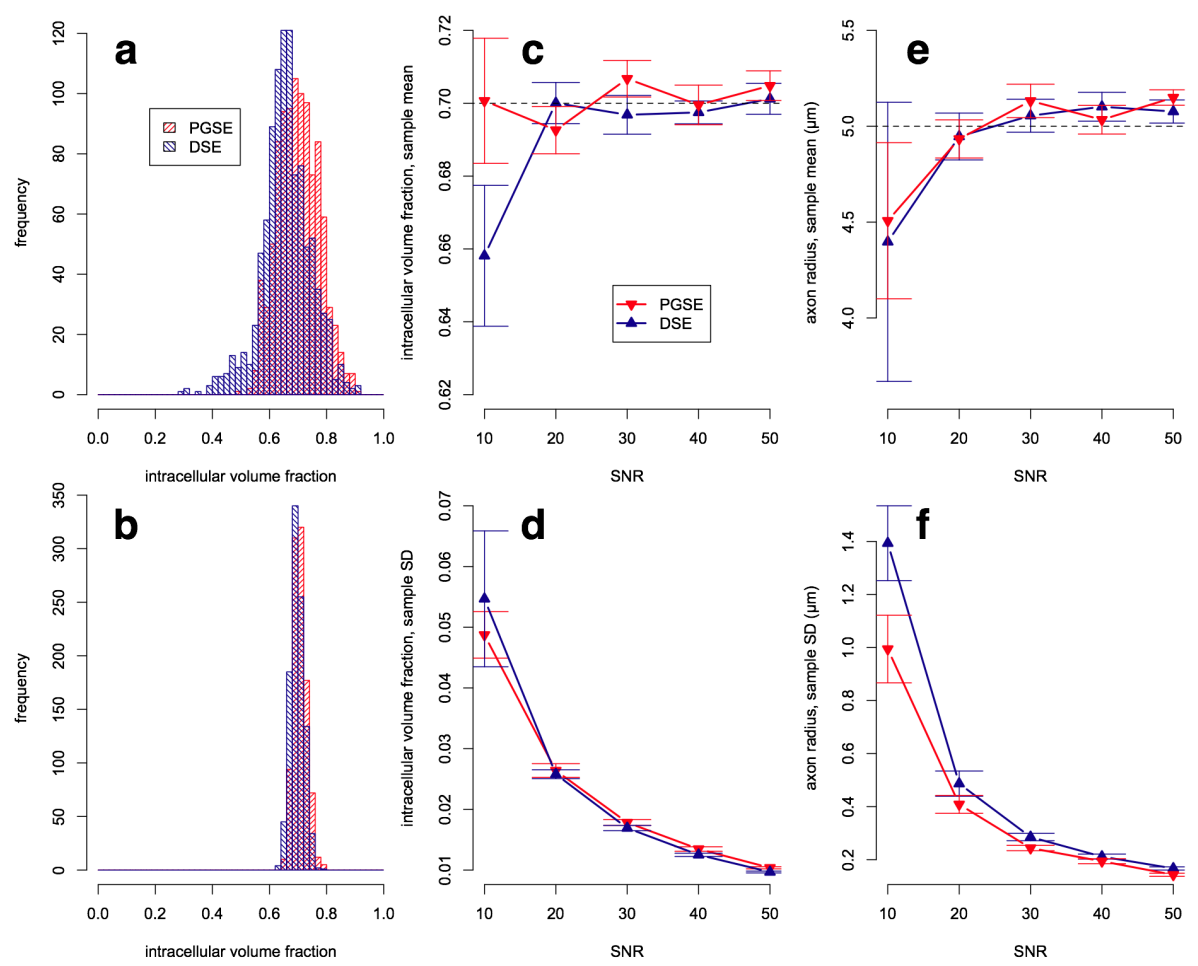

Fig. 3. Results from MCMC sampling of tissue model parameters: histograms of samples from the posterior distribution over $f$ at base SNR 10 (a) and 30 (b), and relationships between SNR and the means and standard deviations of these histograms for $f$ $(\mathrm{c}, \mathrm{d})$ and $R(\mathrm{e}, \mathrm{f})$. Error bars indicate mean \pm standard error over ten fibre directions. Generating values are $f=0.7$ and $R=5 \mu \mathrm{m}$ throughout (dashed lines in c,e). 
sphere [22], are shown in Fig. 2. It can be seen from Fig. 2a that the mean values vary by several orders of magnitude over the five axon radii, but there is little difference between the three pulse sequences. On the other hand, the CVs are slightly smaller for the PGSE case at large $R$, but substantially larger at small $R$, suggesting that the DSE sequence has better orientation invariance when $R$ is small. (This effect persists when the base SNR is higher.) Figure 2c shows the dependence of the cost function value on the azimuthal orientation of the fibre: the spread is much larger for the PGSE sequence at $R=3 \mu \mathrm{m}$, whereas it is almost identical at $R=5 \mu \mathrm{m}$ (not shown). The absolute magnitude of the variance is, however, small in all cases. Data for the constrained DSE sequence is omitted from this subfigure for clarity, but the pattern is very similar to the unconstrained sequence.

Additionally, we investigated the ability of the method to recover tissue parameters from a synthetic data set. We obtain very accurate synthetic signal data using a simulation of 10,000 molecules undergoing Brownian motion, by tracking the phase of each molecule through the optimised sequences to calculate the final signal [23]. The geometric environment in the simulation was the same as in the tissue model, although with uniform diffusivity. Cylinder radius was fixed to $5 \mu \mathrm{m}$ and cylinder separation adjusted to produce an intracellular volume fraction of 0.7. Rician noise was added. We used Markov chain Monte Carlo (MCMC) to sample from posterior distributions over the tissue parameters of interest, using Metropolis-Hastings samplers with zero-mean Gaussian proposal distributions. Variances of these proposal distributions were tuned by hand to give reasonable acceptance rates. Priors were uninformative within appropriate bounds. A burn-in period of 10,000 iterations was used, after which 100 samples were taken with a sampling interval of 1000 iterations. Parameters were initialised with their true values to speed up convergence. This process was repeated for ten generative fibre orientations, $\mathbf{n}$, equally spaced on the sphere. PGSE and unconstrained DSE sequences were tested, and sampling was repeated with protocols optimised using base SNRs of 20, 30, 40 and 50.

Figure 3 shows the results of the MCMC experiments. The sampled posterior distributions over intracellular volume fraction, $f$, a proxy for axon density, are broad at a base SNR of 10 (Fig. 3a); and although both modes are close to the generating value of 0.7 , they differ slightly from one another. By contrast, at a base SNR of 30 (Fig. 3b), both distributions are far tighter. Figure 3c shows that DSE tends to underestimate $f$ at SNR $=10$, although it is generally both more accurate and more stable where SNR $\geq 20$. DSE also has a slight precision advantage at these lower noise levels (Fig. 3d). Both sequences produce underestimates of axon radius, $R$, at low SNR, and their accuracies are largely similar; although PGSE exhibits somewhat greater precision for this parameter over the whole SNR range (Fig. 3e,f). Overall, the differences between the two sequences are small, and neither is consistently superior. 


\section{Discussion}

We have developed the analytical infrastructure needed for active imaging with DSE, facilitating the optimisation of this widely deployed dMRI pulse sequence for estimating the parameters of arbitrarily complex models of living tissue. The DSE sequence has been shown to perform very similarly to PGSE, both in terms of a cost function based on the CRLB, and in terms of its ability to recover generative parameters from simulated data. However, it has a major practical benefit in its reduced sensitivity to eddy currents, which are particularly significant at the large gradient strengths used for experiments of this type. We have shown that eddy current effects can be nulled completely for a particular time constant with little impact on the cost function.

The exact performance of the two dMRI sequences discussed in this paper has been shown to depend on the SNR achievable and the parameter of interest. For the example tissue model we have described, DSE appears to have some advantage for small axon radii. This is probably due to the ability to position pulses next to one another, making effective diffusion times short (cf. Fig. 1b). The noise level is clearly an important factor in performance, and there is a substantial improvement in accuracy and precision in the step from a base SNR of 10 to 20 with both sequences. The latter is quite achievable for in vivo work. We have found no evidence of a consistent noise penalty with DSE, which is sometimes thought to be a problem with the sequence.

A very significant avenue for future work will be the application of DSEbased active imaging to other tissue models and related problems. DSE may prove to be more flexible than PGSE due to the extra degrees of freedom in the parameterisation $(7 M+1$ parameters rather than $3 M)$. We also intend to investigate the effect of eddy currents in acquired images on estimates of tissue model parameters. Implementation of our protocol will be complex due to the substantial number of parameters, but it will be essential to quantify the full benefits of the DSE sequence in active imaging applications.

Acknowledgments: Support for this work was provided through EPSRC grants EP/C536851/1 and EP/E007748. ZN is supported by the Wellcome Trust.

\section{References}

1. Le Bihan, D.: Looking into the functional architecture of the brain with diffusion MRI. Nature Reviews Neuroscience 4 (2003) 469-480

2. Baird, A., Warach, S.: Magnetic resonance imaging of acute stroke. Journal of Cerebral Blood Flow \& Metabolism 18 (1998) 583-609

3. Basser, P., Mattiello, J., Le Bihan, D.: Estimation of the effective self-diffusion tensor from the NMR spin echo. Journal of Magnetic Resonance, Series B 103 (1994) 247-254

4. Horsfield, M., Jones, D.: Applications of diffusion-weighted and diffusion tensor MRI to white matter diseases - a review. NMR in Biomedicine 15 (2002) 570-577

5. Lim, K., Helpern, J.: Neuropsychiatric applications of DTI - a review. NMR in Biomedicine 15 (2002) 587-593 
6. Behrens, T., Johansen-Berg, H., Woolrich, M., Smith, S., Wheeler-Kingshott, C., Boulby, P., Barker, G., Sillery, E., Sheehan, K., Ciccarelli, O., Thompson, A., Brady, J., Matthews, P.: Non-invasive mapping of connections between human thalamus and cortex using diffusion imaging. Nature Neuroscience 6 (2003) 750757

7. Neuman, C.: Spin echo of spins diffusing in a bounded medium. Journal of Chemical Physics 60 (1974) 4508-4511

8. Alexander, D.: Axon radius measurements in vivo from diffusion MRI: a feasibility study. In: Proceedings of the IEEE 11th International Conference on Computer Vision, IEEE (2007)

9. Assaf, Y., Basser, P.: Composite hindered and restricted model of diffusion (CHARMED) MR imaging of the human brain. NeuroImage 27 (2005) 48-58

10. Assaf, Y., Blumenfeld-Katzir, T., Yovel, Y., Basser, P.: Axcaliber: A method for measuring axon diameter distribution from diffusion MRI. Magnetic Resonance in Medicine 59 (2008) 1347-1354

11. Stanisz, G., Szafer, A., Wright, G., Henkelman, R.: An analytical model of restricted diffusion in bovine optic nerve. Magnetic Resonance in Medicine 37 (1997) $103-111$

12. Weng, J.C., Chen, J.H., Kuo, L.W., Wedeen, V., Tseng, W.Y.: Maturationdependent microstructure length scale in the corpus callosum of fixed rat brains by magnetic resonance diffusion-diffraction. Magnetic Resonance Imaging 25 (2007) $78-86$

13. Andersson, J.: Maximum a posteriori estimation of diffusion tensor parameters using a Rician noise model: Why, how and but. NeuroImage 42 (2008) 1340-1356

14. Cercignani, M., Alexander, D.: Optimal acquisition schemes for in vivo quantitative magnetization transfer MRI. Magnetic Resonance in Medicine 56 (2006) 803-810

15. MacKay, D.: Information-based objective functions for active data selection. Neural Computation 4 (1992) 590-604

16. Alexander, D.: A general framework for experiment design in diffusion MRI and its application to measuring direct tissue-microstructure features. Magnetic Resonance in Medicine 60 (2008) 439-448

17. Stejskal, E., Tanner, J.: Spin diffusion measurements: spin echoes in the presence of a time-dependent field gradient. Journal of Chemical Physics 42 (1965) 288-292

18. Reese, T., Heid, O., Weisskoff, R., Wedeen, V.: Reduction of eddy-current-induced distortion in diffusion MRI using a twice-refocused spin echo. Magnetic Resonance in Medicine 49 (2003) 177-182

19. van Gelderen, P., DesPres, D., van Zijl, P., Moonen, C.: Evaluation of restricted diffusion in cylinders. Phosphocreatine in rabbit leg muscle. Journal of Magnetic Resonance, Series B 103 (1994) 255-260

20. Heid, O.: Eddy current-nulled diffusion weighting. In: Proceedings of the ISMRM 8th Scientific Meeting \& Exhibition, International Society for Magnetic Resonance in Medicine (2000) 799

21. Henkelman, R.: Measurement of signal intensities in the presence of noise in MR images. Medical Physics 12 (1985) 232-233

22. Rakhmanov, E., Saff, E., Zhou, Y.: Minimal discrete energy on the sphere. Mathematical Research Letters 1 (1994) 647-662

23. Hall, M., Alexander, D.: A tissue model of white matter undergoing tissue swelling. In: Proceedings of the MICCAI Workshop on Computational Diffusion MRI. (2008) 